\title{
Influence of the Long-Range RKKY Interaction on a Formation of Magnetization Plateaus in the Generalised Ising Model on the Shastry-Sutherland Lattice
}

\author{
L. Regeciová* AND P. FARKAŠOVSKÝ \\ Institute of Experimental Physics, SAS, Watsonova 47, 04001 Košice, Slovakia
}

\begin{abstract}
We present a simple model for a description of magnetization processes in metallic rare-earth tetraborides. It is based on the two-dimensional Ising model, in which two spins on the Shastry-Sutherland lattice interact via the long-range RKKY interaction mediated by conduction electrons. The model is solved by a combination of the standard Metropolis algorithm and the parallel tempering method and it yields the rich spectrum of magnetic solutions (magnetization plateaus), depending on the value of the Fermi momentum $k_{F}$ and the external magnetic field $h$. In particular, we have found the following set of individual magnetization plateaus with fractional magnetization $m / m_{s}=1 / 12,1 / 6,1 / 4,1 / 3,3 / 8,5 / 12,1 / 2,7 / 12,2 / 3$, which for different values of $k_{F}$, form various sequences of plateaus, changing from very complex ones, appearing near the points $k_{F}=2 \pi / 1.243$ to very simple ones appearing away from this point. The importance of these results for a description of real rare-earth tetraborides is discussed.
\end{abstract}

DOI: 10.12693/APhysPolA.137.625

PACS/topics: magnetization plateaus, Shastry-Sutherland lattice, RKKY interaction

\section{Introduction}

The metallic rare-earth tetraborides with the unique two-dimensional structure topologically equivalent to the Shastry-Sutherland lattice (SSL) are known to host a rich variety of physical phenomena among which the appearance of fascinating sequences of magnetization plateaus with the fractional magnetization (FM) has attracted the greatest attention. The SSL [1] can be described as a square lattice with antiferromagnetic couplings $J_{1}$ and additional antiferromagnetic couplings $J_{2}$ between next nearest neighbours (NN) in every second square (in rare-earth tetraborides $J_{1}=J_{2}$ ). In particular, for $\mathrm{ErB}_{4}$ the $\mathrm{FM}$ plateaus have been found at $m / m_{s}=1 / 2[2]$, for $\mathrm{HoB}_{4}$ at $m / m_{s}=1 / 3,4 / 9$, and $3 / 5$ [2], for $\mathrm{TbB}_{4}$ at $m / m_{s}=2 / 9,1 / 3,4 / 9,1 / 2$, and $7 / 9[3]$ and for $\mathrm{TmB}_{4}$ at $m / m_{s}=1 / 11,1 / 9,1 / 7$, and $1 / 2[4]$. These compounds have a large total magnetic moment and thus they can be considered as classical spin systems. The antiferromagnetic Ising model (IM) and its extensions [5-7] on the SSL were able to describe, at least partially, some of the experimentally observed sequences of magnetization plateaus with the FM. However, spin models ignore completely the metallic nature of these compounds. Some theoreticians speculate that for the correct description of magnetization processes in rare-earth tetraborides it is necessary to take into account both the spin and electron subsystems as well as

*corresponding author; e-mail: regeciova@saske.sk the interaction between them. In particular, a previous work [8] has showed that the model based on the coexistence of both subsystems has a great potential to describe complete sequences of magnetization plateaus observed in some rare-earth tetraborides, e.g., $\mathrm{TmB}_{4}$.

\section{Model}

In a recent paper, Feng et al. [9] have introduced an alternative model, which takes into account the long-range interaction, as well as the presence of conduction electrons. Specifically, the IM with the Ruderman-KittelKasuya-Yosida (RKKY) interaction, which can be defined as coupling between localized inner electron spins in a metal by means of an interaction through the conduction electrons, was studied numerically and various magnetization plateaus, depending on the value of the Fermi momentum $k_{\mathrm{F}}$ are confirmed. In particular, for $k_{\mathrm{F}}=2 \pi / 1.243$, the case corresponding to the $\mathrm{TmB}_{4}$ compound, they found two magnetization plateaus with FM, and namely, the $1 / 4$ and $1 / 2$ plateaus. However, these results contradict other theoretical results [7] obtained on the IM with the additional NN interaction on which this model can be mapped. Indeed, for $k_{\mathrm{F}}=2 \pi / 1.243$, the IM with RKKY interaction can be mapped on the standard IM with the first, second, third, and fourth NN interaction by putting [9]: $J_{1}=J_{2}=1, J_{3}=0.1135$, and $J_{4}=-0.0771$. However, for these values, besides the $1 / 2$ plateau, the standard IM predicts also the wide $1 / 3$ plateau [7], which is completely absent in the solution obtained by Feng et al. [9]. This discrepancy creates doubts about also other results presented in this work, in particularly the full $h-k_{\mathrm{F}}$ phase diagram. 
For this reason we have decided to re-examine carefully the same model using the same method, the standard Metropolis algorithm supported by the parallel tempering method [7], and the same conditions (periodic boundary conditions and the cut-off radius of RKKY $r=6$ ) as in [9]:

$$
H=\sum_{i, j} J_{i j} S_{i}^{z} S_{j}^{z}-h \sum_{i} S_{i}^{z},
$$

where variable $S_{i}^{z}$ denotes the Ising spin on the site $i$ and $h$ is the magnetic field. The matrix elements $J_{i j}$ of the RKKY interaction coupling between two spins localized at sites $i$ and $j$ can be written as

$$
J_{i j}=-J_{0} a^{3}\left[\frac{\cos \left(2 k_{\mathrm{F}} r_{i j}\right)}{r_{i j}^{3}}-\frac{\sin \left(2 k_{\mathrm{F}} r_{i j}\right)}{2 k_{\mathrm{F}} r_{i j}^{4}}\right],
$$

where $a$ is the lattice constant, $r_{i j}$ is the distance between the sites $i$ and $j$ on the real lattice, $k_{F}$ is Fermi momentum, and $J_{0}$ denotes the strength of the RKKY interaction.

\section{Results and discussion}

To verify the convergence of the Monte Carlo results we have started our numerical studies on the finite cluster of $L=6 \times 6$, where the exact numerical results are also accessible (Fig. 1a). A comparison of the exact magnetization curve calculated for $T=0$ and the Monte Carlo one obtained for $T=0.02$ shows that selected temperature $T=0.02$ is sufficient to approximate reliably the groundstate properties of the model. These calculations exhibit, besides the $1 / 2$ plateau and the small $1 / 6$ plateau, also the large $1 / 3$ plateau which is completely absent on the magnetization curve obtained by Feng et al. [9]. Since the size of cluster used in our simulations is relatively small, we have performed the systematic study of the model on larger lattices. The results of Monte Carlo simulations obtained on the $L=12 \times 12$ cluster (inset in Fig. 1a) clearly demonstrate that the $1 / 3$ plateau still persists on the magnetization curve, although its width is reduced in comparison the $L=6 \times 6$ cluster. In addition, we have found also the $7 / 18$ plateau which indicates that the magnetization curve will have a more complex structure than the one described in work [9], where only the $1 / 4$ and $1 / 2$ plateaus are observed. This conjecture supports also the results obtained on the $L=24 \times 24$ cluster (Fig. 1b), where, besides these two plateaus, we have found additional plateaus with $m / m_{s}=29 / 96,1 / 3,13 / 36,3 / 8$, and $55 / 144$.

Absence of these plateaus on the Feng magnetization curve rises the question regarding which results are right. To answer this question we have directly calculated the ground state energies $\left(E_{0}^{f}, E_{1 / 4}^{f}, E_{1 / 2}^{f}, E_{1}^{f}\right)$ for all phases that were identified by Feng et al. [9] on the magnetization curve and compare them with the ground state energy $\left(E_{1 / 3}\right)$ of the $\mathrm{A}(1 / 3)$ plateaus phase identified on our magnetization curve for wide range of $h$ values (see Fig. 2a). This comparison shows that our $\mathrm{A}(1 / 3)$ plateau phase suppresses completely the stability region
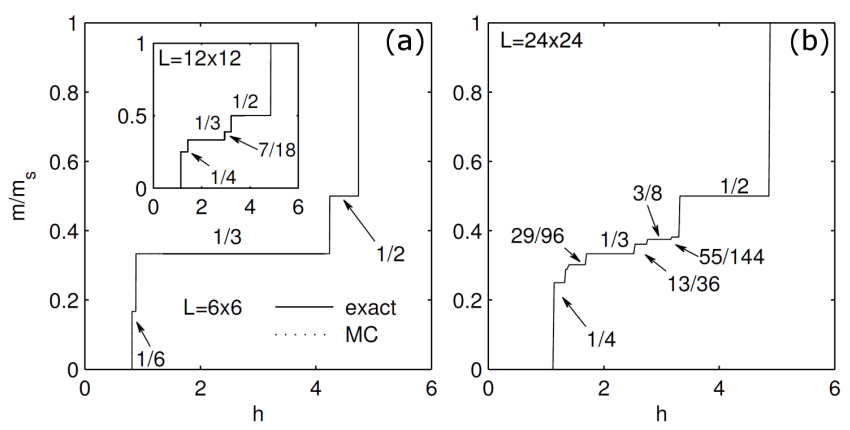

Fig. 1. The magnetization curve of the IM with RKKY interaction calculated on the $L=6 \times 6$ cluster (a), the $L=12 \times 12$ cluster (inset) and the $L=24 \times 24$ cluster (b).
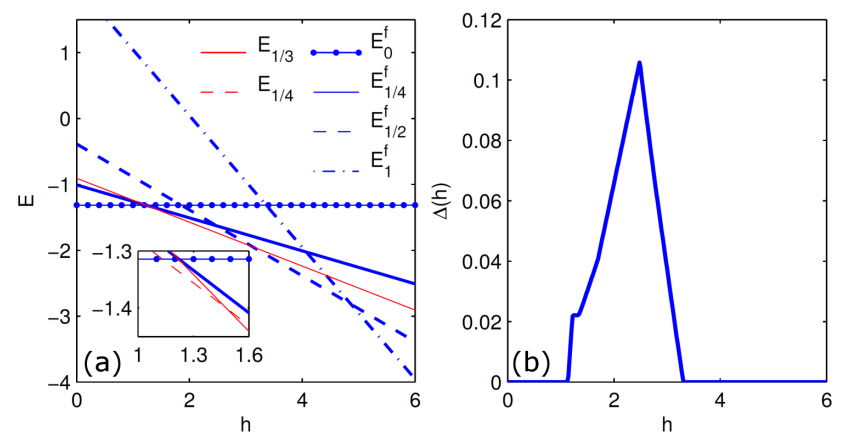

Fig. 2. (a) The ground-state energies corresponding to different plateau phases identified on Feng's and our magnetization curve. The inset compares energies of the Feng and our $1 / 4$ plateau phase. (b) The difference $\Delta(h)$ between the Feng lowest energy (calculated for all phases from their magnetization curve at given $h$ ) and our one.

of the Feng $1 / 4$ plateau phase and reduces partially the stability region of $1 / 2$ plateau phase. The appearance of the $1 / 4$ plateau on our magnetization curve is obviously the consequence of the fact that our $\mathrm{A}(1 / 4)$ plateau phase has lower ground-state energy and thus $E_{1 / 4}<E_{1 / 4}^{f}$ (inset of Fig. 2a). In Fig. 2b we plot the difference $\Delta(h)=E_{\min }^{f}(h)-E_{\min }(h)$ between the Feng lowest energy $E_{\min }^{f}(h)$ and our one, $E_{\min }(h)$, which clearly shows that our phases identified in the central part of the magnetization curve have considerably lower energies than ones found by Feng et al. [9].

These results raise the question about the reliability of magnetization curves obtained in [9] for other values of the Fermi momentum $k_{\mathrm{F}}$. For this reason we have also re-examined the full $k_{\mathrm{F}}-h$ phase diagram of the model (Fig. 3). We have found that both phase diagrams accord qualitatively for $k_{\mathrm{F}}$ below the unphysical region, where we have found the same plateau phases except the $q$-Néel phase $\mathrm{A}(0)$ which is replaced by the more complex antiferromagnetic phase $\mathrm{C}(0)$. However, they completely differ in the opposite limit. Above the 


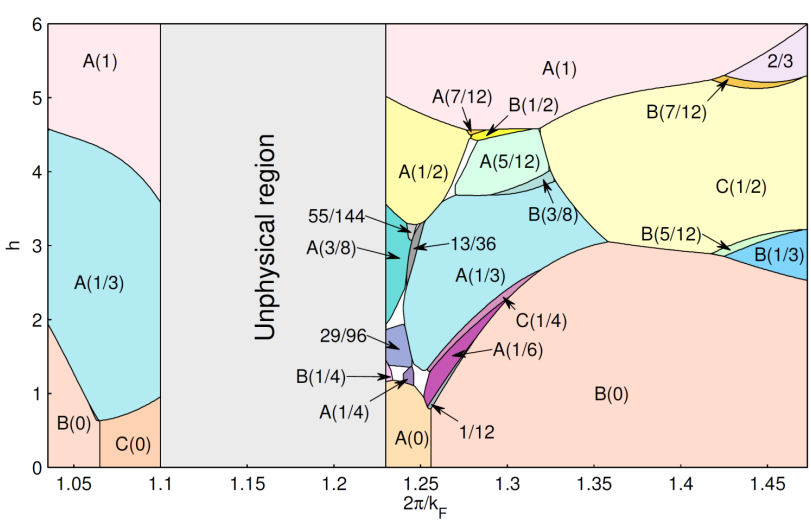

Fig. 3. The magnetic phase diagram of the model in the $k_{\mathrm{F}}-h$ plane calculated for the $L=24 \times 24$ cluster.

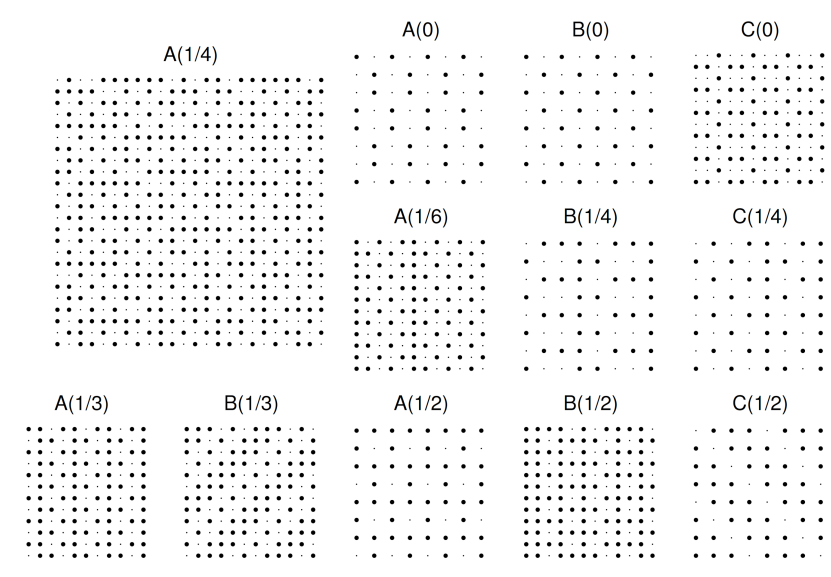

Fig. 4. Selected spin configurations that enter to the $k_{\mathrm{F}}-h$ phase diagram in Fig. 3 obtained by standard Metropolis algorithm.

unphysical region Feng et al. found seven macroscopic phases: besides the Néel $\mathrm{B}(0)$, $q$-Néel $\mathrm{A}(0)$, and ferromagnetic $\mathrm{A}(1)$ phases there are also four ferrimagnetic phases with $m / m_{s}=1 / 6,1 / 4,5 / 12$ and $1 / 2$.

Our Monte Carlo results show that the phase diagram has a more complex structure. The main difference between our and Feng's diagrams consists in the appearance of the large $\mathrm{A}(1 / 3)$ phase in the central part of our phase diagram. The second one is the observation that the Néel phase $\mathrm{B}(0)$ extends to high values of $h$ in comparison to results of [9] and almost completely suppresses the phase $\mathrm{A}(1 / 6)$. The third one is the appearance of some plateau phases in two or even three different spin arrangements. For example we have found two different spin arrangements for the $1 / 3$ plateau and three different spin arrangements for the $1 / 4$ and $1 / 2$ plateau (see Fig. 4). Generally, our phase diagram is much richer in comparison to one presented by Feng et al. and in addition to their phases, it is able to describe also the existence of some other phases with FM like: $m / m_{s}=1 / 12,3 / 8$, $7 / 12$, and $2 / 3$.

\section{Conclusions}

We have re-examined the magnetic phase diagram of the IM with the long-range RKKY interaction on the SSL. It is shown that our results improve considerably the results obtained by Feng et al. In particular, we have found the following set of individual magnetization plateaus with $\mathrm{FM}: m / m_{s}=1 / 12,1 / 6,1 / 4,1 / 3,3 / 8$, $5 / 12,1 / 2,7 / 12$, and $2 / 3$, which, for different values of $k_{\mathrm{F}}$, form various sequences of plateaus. Since the change of $k_{\mathrm{F}}$ can be induced by doping, the model is able to predict the complete sequences of magnetization plateaus, that could appear in the tetraboride solid solution.

\section{Acknowledgments}

This work was supported by projects VEGA 2-0112-18, APVV-17-0020, ITMS 2220120047, ITMS 26230120002, and IMTS 26210120002.

\section{References}

[1] B.S. Shastry, B. Sutherland, Physica $B+C$ 108, 1069 (1981).

[2] S. Mataš, K. Siemensmeyer, E. Wheeler, et al., J. Phys. Conf. Ser. 200, 032041 (2010).

[3] S. Yoshi, Y. Tamamoto, M. Hagiwara, S. Michumura, A. Shigekawa, F. Iga, T. Takabatake, K. Kindo, Phys. Rev. Lett. 101, 087202 (2008).

[4] K. Siemensmeyer, E. Wulf, H.K. Mikeska, K. Flachbart, S. Gabáni, S. Mataš, P. Priputen, A. Efdokimova, N. Shitsevalova, Phys. Rev. Lett. 101, 177201 (2008).

[5] M.C. Chang, M.F. Yang, Phys. Rev. B 79, 104411 (2009).

[6] Y. Dublenych, Phys. Rev. E 88, 022111 (2013).

[7] P. Farkašovský, L. Regeciová, Eur. Phys. J. B 92, 33 (2019).

[8] P. Farkašovský, H. Čenčariková, S. Mataš, Phys. Rev. B 82, 54410 (2010).

[9] J.J. Feng, L. Huo, W.C. Huang, Y. Wang, M.H. Qin, J.M. Liu, Z. Ren, EPL 105, 17009 (2014). 